\title{
Vitamin $D$, calcium and dairy intake, and risk of oesophageal adenocarcinoma and its precursor conditions
}

\author{
Helen G. Mulholland*, Liam J. Murray, Lesley A. Anderson and Marie M. Cantwell on behalf of the \\ FINBAR study group \\ Cancer Epidemiology and Health Services Research Group, Centre for Public Health, Queens University Belfast, Mulhouse \\ Building, RVH Site, Grosvenor Road, Belfast BT12 6BJ, UK
}

(Received 9 August 2010 - Revised 25 January 2011 - Accepted 26 January 2011 - First published online 9 May 2011)

\begin{abstract}
Evidence is accumulating that vitamin D may be protective against carcinogenesis, although exceptions have been observed for some digestive tract neoplasms. The aim of the present study was to explore the association between dietary vitamin D and related nutrients and the risk of oesophageal adenocarcinoma and its precursor conditions, Barrett's oesophagus and reflux oesophagitis. In an all-Ireland case-control study conducted between March 2002 and July 2005, 218 oesophageal adenocarcinoma patients, 212 Barrett's oesophagus patients, 208 reflux oesophagitis patients and 252 population-based controls completed a 101-item FFQ, and provided lifestyle and demographic information. Multiple logistic regression analysis was applied to examine the association between dietary intake and disease risk. Oesophageal adenocarcinoma risk was significantly greater for individuals with the highest compared with the lowest tertile of vitamin $\mathrm{D}$ intake (OR 1.99, 95\% CI 1.03, 3.86; $P$ for trend=0.02). The direct association could not be attributed to a particular vitamin D food source. Vitamin D intake was unrelated to Barrett's oesophagus and reflux oesophagitis risk. No significant associations were observed for Ca or dairy intake and oesophageal adenocarcinoma, Barrett's oesophagus or reflux oesophagitis development. High vitamin D intake may increase oesophageal adenocarcinoma risk but is not related to reflux oesophagitis and Barrett's oesophagus. Ca and dairy product intake did not influence the development of these oesophageal lesions. These findings suggest that there may be population subgroups at an increased risk of oesophageal adenocarcinoma if advice to improve vitamin D intake from foods is implemented. Limited work has been conducted in this area, and further research is required.
\end{abstract}

Key words: Diet: Vitamin D: Reflux oesophagitis: Barrett's oesophagus: Oesophageal adenocarcinoma

Reflux oesophagitis and Barrett's oesophagus represent early transitional stages in the progression of normal oesophageal tissue to oesophageal adenocarcinoma, arising from the repetitive reflux of gastric acid and bile. This spectrum of oesophageal disorders is of growing concern in developed nations. Although relatively rare, the widely reported rise in oesophageal adenocarcinoma incidence in the UK has continued into the new millennium ${ }^{(1)}$. A systematic analysis of time trends has also confirmed that the prevalence of both gastrooesophageal reflux disease and oesophagitis has increased since the 1980s, particularly in North America and Europe ${ }^{(2)}$. Although Barrett's oesophagus is a designated premalignant condition for oesophageal adenocarcinoma ${ }^{(3)}$, progression along the carcinogenic pathway is selective, with approximately $0.5 \%$ of patients going on to develop this malignancy $^{(4-6)}$. Such selective development has generated interest in identifying modifiable risk factors for cancer progression in this small subsample of patients.
Despite certain dietary triggers such as fat, chocolate, mints, onions, citrus fruit and tomatoes being observed for reflux episodes, there is little evidence to suggest that these play a role in the subsequent development of oesophageal adenocarcinoma ${ }^{(7)}$. Only a limited number of studies have explored diet as an aetiological agent for these oesophageal lesions, with studies to date largely focusing on fruit and vegetable, macronutrient and antioxidant vitamin intake ${ }^{(8,9-13)}$.

There is considerable evidence in the scientific literature citing a protective role for vitamin $\mathrm{D}$ against the development of many cancers ${ }^{(14)}$. Vitamin D has been shown to have several generic anti-carcinogenic effects such as suppressing cell proliferation, promoting cell differentiation and regulating apoptosis $^{(15)}$. Consistent inverse associations are evident for optimum vitamin D status and colorectal cancer and adenoma risk in particular ${ }^{(16,17)}$, and such findings are fuelling momentum for randomised controlled trials of vitamin D supplementation in cancer prevention. However, the hypothesised

Abbreviation: FINBAR, Factors INfluencing the Barrett's Adenocarcinoma Relationship.

* Corresponding author: Dr H. G. Mulholland, fax + 442890235900 , email h.mulholland@qub.ac.uk 
protective effects of vitamin $\mathrm{D}$ in cancer prevention may not be universal. There have been notable exceptions to this protective relationship though from studies of vitamin $\mathrm{D}$ and other digestive tract neoplasms ${ }^{(18)}$. Increased risks of oesophageal squamous cell carcinoma and its precursor, squamous dysplasia of the oesophagus, have been demonstrated for individuals with the highest serum concentrations of 25-hydroxyvitamin D in a Chinese population ${ }^{(19,20)}$.

Vitamin D and Ca are highly related in physiological terms, with low serum Ca status known to induce the synthesis of the active form of vitamin $\mathrm{D}$ from 25-hydroxyvitamin $\mathrm{D}$ in the kidney $^{(21)}$. Adequate Ca consumption has been demonstrated to reduce the risk of colorectal cancer, although reports of its relationship with the risk of other cancers have been inconsistent ${ }^{(12)}$. These equivocal findings are suggested to be due to confounding from high fat contents of common food sources, particularly dairy products.

The most recent World Cancer Research Fund global report on food, nutrition, physical activity and the prevention of cancer could not draw any conclusions about the association of vitamin $\mathrm{D}$, Ca or dairy product intake with oesophageal cancer risk due to a lack of available evidence ${ }^{(12)}$. Furthermore, no studies to date have investigated the association between vitamin $\mathrm{D}$, Ca or dairy intake and the risk of the precursor conditions, reflux oesophagitis and Barrett's oesophagus.

The aim of the present investigation was to explore the association between vitamin D, Ca and dairy intake and the risk of reflux oesophagitis, Barrett's oesophagus and oesophageal adenocarcinoma in an all-Ireland case-control study.

\section{Methods}

\section{Study design}

The Factors INfluencing the Barrett's Adenocarcinoma Relationship (FINBAR) study is an all-Ireland populationbased case-control study that was established to investigate the aetiology of reflux oesophagitis, Barrett's oesophagus and oesophageal adenocarcinoma, as described previously $^{(9,13,22)}$. Incident cases of oesophageal adenocarcinoma ( $n$ 227) and long-segment Barrett's oesophagus ( $n$ 224), and normal population controls ( $n$ 260) were recruited throughout the island of Ireland between March 2002 and July 2005. Reflux oesophagitis cases ( $n$ 230) were recruited in Northern Ireland only. Barrett's oesophagus, reflux oesophagitis and control subjects were frequency matched within 5-year age and sex strata to the distribution of oesophageal adenocarcinoma patients, up to a maximum age of 85 years. The present study was conducted according to the guidelines laid down in the Declaration of Helsinki, and all procedures involving human subjects/patients were approved by the Research Ethics Committee of the Queen's University Belfast (Northern Ireland); Clinical Research Ethics Committee of Cork Teaching Hospitals; and Research Ethics Committee Board of St James's Hospital (Dublin, Ireland). Written informed consent was obtained from all subjects.

\section{Study participants}

Incident cases of histologically confirmed oesophageal adenocarcinoma were identified from hospital clinical records in the Republic of Ireland or via electronic pathology records in Northern Ireland. Patients with endoscopically diagnosed long-segment Barrett's oesophagus ( $\geq 3 \mathrm{~cm}$ in length) were recruited if the presence of specialised intestinal metaplasia had been histologically confirmed. Patients with dysplasia were excluded. Reflux oesophagitis patients were included if they had been diagnosed with mucosal erosions in the oesophagus, classified as grades 2-4 or grades B, C or D using the Savary Miller/Hetzel-Dent or Los Angeles methods ${ }^{(23)}$, respectively, at the time of endoscopy. Population-based controls were adults with no previous history of Barrett's oesophagus, oesophageal or other gastrointestinal cancers. Controls were recruited at random in Northern Ireland via the General Practice Master Index and in the Republic of Ireland from four General Practices representing urban and rural areas (Dublin and Cork). The response rates of eligible participants ranged from $42 \%$ for controls to 69,82 and $74 \%$ for reflux oesophagitis, Barrett's oesophagus and oesophageal adenocarcinoma cases, respectively.

\section{Data collection}

Trained interviewers collected data from study participants using an electronic questionnaire. Dietary intake was assessed using a 101-item FFQ, adapted for the Irish population from a version of the European Prospective Investigation into Cancer and Nutrition $\mathrm{FFQ}^{(24)}$, by incorporating foods reported as commonly eaten in the North/South Ireland Food Consumption Survey ${ }^{(25)}$. The FFQ did not specifically ask for information on vitamin D or Ca supplement usage. Mean daily dietary intakes were calculated from the FFQ using Q-Builder (Tinuviel Software, Llanfechell, Anglesey, UK). Participants were asked to recall their dietary habits over the 12-month period 5 years before the interview. BMI 5 years before the interview was calculated using self-reported weight $(\mathrm{kg})$ divided by current height $\left(\mathrm{m}^{2}\right)$, as measured by the interviewer. Helicobacter pylori infection status was assessed from serum samples using a Western blot assay, as described previously $^{(26)}$.

\section{Statistical analysis}

A total of twenty-two study participants failed to provide any dietary information, and information on dairy product intake was missing for a further twenty-nine participants, and therefore these individuals were excluded from the present analysis. This equated to twenty-two reflux oesophagitis cases, twelve Barrett's oesophagus cases, nine oesophageal adenocarcinoma cases and eight controls being excluded, leaving 208 reflux oesophagitis patients, 212 Barrett's oesophagus patients, 218 oesophageal adenocarcinoma patients and 252 population controls for consideration in this analysis. The comparison group for all analysis exploring reflux oesophagitis risk was restricted to controls recruited in Northern Ireland, 
since reflux oesophagitis patients were recruited in Northern Ireland only.

Key characteristics and mean nutrient intakes of cases in relation to controls were examined using independent $t$ tests for continuous variables and $\chi^{2}$ tests for categorical variables. Unconditional multiple logistic regression analysis was utilised to explore the association between vitamin D, Ca and dairy intake and disease risk by tertiles of intake, resulting in OR and corresponding $95 \%$ CI. In order to test for trend, each individual within a particular tertile was assigned the median intake value for that tertile before inclusion in the regression model. In multivariate-adjusted models, confounding variables were age (years), sex, energy intake $(\mathrm{kJ} / \mathrm{d})$, smoking status (current/previous/never), education (years), BMI 5 years before the interview $\left(\mathrm{kg} / \mathrm{m}^{2}\right)$, occupation (manual/nonmanual), alcohol intake (g/d), regular non-steroidal inflammatory drug use (weekly use for at least 6 months' duration), H. pylori infection (seronegative/seropositive), energyadjusted glycaemic index intake (units/4184kJ per d), energy-adjusted saturated fat intake $(\mathrm{g} / 4184 \mathrm{~kJ}$ per $\mathrm{d})$ and location (Northern Ireland/Republic of Ireland). Reflux oesophagitis analyses were restricted to Northern Ireland controls only. Covariates were chosen based on previously known risk factors for oesophageal lesions. In a separate multivariate-adjusted regression model, we further tested for regular gastro-oesophageal reflux symptoms (ever/never), since it is debatable whether reflux symptoms may confound or be on the causal pathway between disease risk and the dietary variables of interest. Stratified analyses were conducted according to BMI categories, smoking and H. pylori infection status, which were further adjusted for each other, and the likelihood ratio test was applied to evaluate potential interactions. Statistical analysis was performed using Intercooled STATA version 9.2 (StataCorp, College Station, TX, USA).

\section{Results}

Table 1 summarises the key characteristics and dietary variables of interest for all three case groups and their respective controls. Reflux oesophagitis and Barrett's oesophagus cases did not differ from their respective controls by daily energy, vitamin $\mathrm{D}, \mathrm{Ca}$ or dairy product intakes. In contrast, oesophageal adenocarcinoma cases had significantly higher energy and vitamin D intakes compared with controls, although $\mathrm{Ca}$ and dairy intakes were similar between the groups. Vitamin $\mathrm{D}$ and $\mathrm{Ca}$ intakes represent intakes from foods only and do not include intakes from supplements, which we were unable to assess.

As illustrated in Table 2, Ca intake was inversely associated with odds of reflux oesophagitis when comparing the highest with the lowest tertile of intake (OR 0.37, $95 \%$ CI $0 \cdot 17,0 \cdot 80$; $P$ for trend $=0.01)$ in age- and energy-adjusted regression analysis. However, no significant associations were identified for $\mathrm{Ca}$, vitamin $\mathrm{D}$ or total dairy product intake and reflux oesophagitis risk once the adjusted for potential confounders.

No significant associations were found between vitamin D or Ca intakes and Barrett's oesophagus risk either before or after multivariate adjustments, as shown in Table 2. There was also little evidence of an association between dairy product consumption and odds of Barrett's oesophagus in multivariate-adjusted regression analysis. Once the analysis was further adjusted for gastro-oesophageal reflux symptoms, however, a significant positive association emerged, indicating a nearly twofold increased risk of Barrett's oesophagus for individuals with the highest tertile of dairy product intake.

A significant direct association was observed between the highest tertile $v$. the lowest tertile of vitamin $\mathrm{D}$ intake and odds of oesophageal adenocarcinoma, even after adjustment for confounders (OR 1.99, 95\% CI 1.03, 3.86; $P$ for trend $=0.02$ ). This was attenuated slightly after adjustment for gastro-oesophageal reflux symptoms, but a significant trend remained ( $P$ for trend $=0.03$ ). Consumption of dairy products also tended to be positively associated with oesophageal adenocarcinoma risk, although this did not achieve statistical significance until multivariate-adjusted analyses further accounted for gastro-oesophageal reflux symptoms (OR 1.89, 95\% CI 1.02, 3.50; $P$ for trend=0.04). No significant relationship was found between dietary $\mathrm{Ca}$ and oesophageal adenocarcinoma risk (Table 2).

To investigate the unexpected direct association observed between vitamin $\mathrm{D}$ and oesophageal adenocarcinoma risk further, we explored intake of foods commonly consumed in the Irish diet that are rich sources of vitamin D. Oily fish, egg, margarine and total red meat intakes were not significantly associated with oesophageal adenocarcinoma risk and could not explain the association observed (Table 3). Individuals who consumed liver were at heightened odds of oesophageal adenocarcinoma compared with non-consumers after adjusting for potential confounders (OR 1.73, 95\% CI $1 \cdot 01,2 \cdot 97)$. However, further adjustment for liver intake actually strengthened, rather than attenuated, the positive association between vitamin $\mathrm{D}$ and oesophageal adenocarcinoma risk (OR 1.99, 95\% CI 1.03, 3.86) when comparing extreme categories of intake. Furthermore, individuals who consumed liver also had elevated odds of Barrett's oesophagus compared with non-consumers (OR 1·77, 95\% CI 1·06, 2.98), thus failing to demonstrate the specificity to oesophageal adenocarcinoma that was shown by the results of vitamin D. Additional adjustment for total vitamin A intake could not explain the deleterious effect of liver, although it was not possible to investigate the effects of retinyls and pro-vitamin A carotenoids separately. It should be noted that unlike in the USA, milk is not fortified with vitamin D in either Northern Ireland or the Republic of Ireland and was therefore not considered in the vitamin D-related food analysis.

When analysis was stratified by BMI categories, as shown in Table 4, a 2.5-fold elevated OR for oesophageal adenocarcinoma was limited to overweight and obese individuals with the highest category of vitamin D intake, and was not observed in normal-weight individuals. The direct association between vitamin $\mathrm{D}$ and oesophageal adenocarcinoma risk also appeared to be modified by $H$. pylori status, and was only evident in individuals with the highest intakes who were $H$. pylori-positive (OR 3.78, $95 \%$ CI 1.44, 9.91; $P$ for trend $=0.008$ ) and not $H$. pylori-negative cases (OR 0.98, $95 \%$ CI $0.35,2.77 ; P$ for trend $=0.72$ ). Ever smokers were 
Table 1. Characteristics and nutrient intakes of reflux oesophagitis, Barrett's oesophagus and oesophageal adenocarcinoma patients and controls (Mean values, standard deviations, number of patients and percentages)

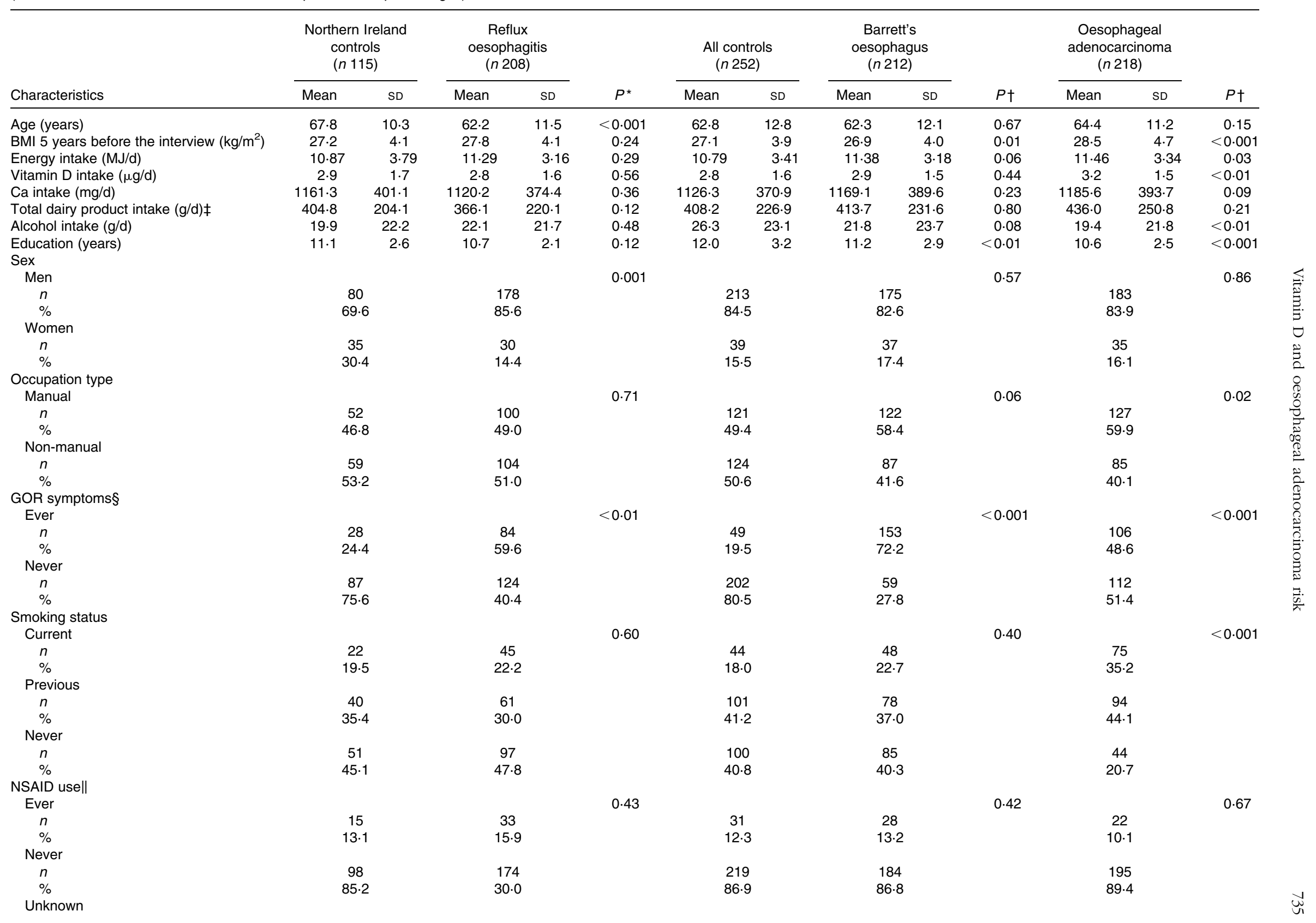


also at increased odds of oesophageal adenocarcinoma with increasing vitamin $\mathrm{D}$ intakes, which appeared to be largely due to previous smoking habits. Notably though, all formal tests for the interaction between vitamin D and these stratified factors failed to reach statistical significance.

\section{Discussion}

Findings from this population-based case-control study indicate that high vitamin $\mathrm{D}$ intakes from foods are associated with elevated oesophageal adenocarcinoma risk but are unlikely to influence earlier stages of the carcinogenic pathway. Ca intake was not related to the development of any of the oesophageal lesions investigated. The association between dairy product intake and Barrett's oesophagus and oesophageal adenocarcinoma risk only became significant once analyses were further adjusted for gastro-oesophageal reflux symptoms. This would suggest that individuals are consuming dairy products to soothe their reflux symptoms, rather than being associated with the aetiology of these conditions.

The present findings that high vitamin D intake increases oesophageal adenocarcinoma risk are stronger than those of a US population-based case-control study, which identified a non-significant $10 \%$ increased risk of oesophageal adenocarcinoma comparing the 75 th centile with the 25 th centile of intake ${ }^{(27)}$. Findings from the same study corroborate ours, in that $\mathrm{Ca}$ intake was also unrelated to oesophageal adenocarcinoma risk; however, an increased risk was identified for those individuals with high-fat dairy intake ${ }^{(27,28)}$. No such connection was observed for low-fat dairy intake, and so the authors have concluded that fat content was responsible for the elevated oesophageal adenocarcinoma risk ${ }^{(28)}$. We were unable to disentangle low- and high-fat dairy product intake in the present study; however, all analyses were adjusted for saturated fat intake.

A biologically plausible mechanism for the overall direct association between dietary vitamin $\mathrm{D}$ and oesophageal adenocarcinoma risk can only be speculated. Undoubtedly, the evidence for anti-carcinogenic properties of vitamin D in the body ${ }^{(29)}$ far outweighs that describing an opposing role. Specific evidence to explain an increased risk of oesophageal malignancy is also lacking. A feeding study of a tumorigenic diet administered to mice in addition to vitamin $\mathrm{D}$ was found to have no significant effects in oesophagus tissue ${ }^{(30)}$. To fully probe the association, we first investigated common sources of vitamin D in the Irish diet to explore whether there was a nefarious effect of a particular food. Only liver was directly related to oesophageal adenocarcinoma risk; however, further adjustment for liver intake only served to strengthen the association observed for vitamin D. It is therefore unlikely that an alternative carcinogenic agent in vitamin D food sources is responsible for the association observed.

We then performed stratified analysis to determine whether the direct association was limited to a certain subgroup of individuals. The increased risk was heightened among overweight individuals, $H$. pylori-positive individuals and ever, particularly past, smokers, although interactions between these factors and vitamin D intake were not significant. 
Table 2. Vitamin D, calcium and dairy product intake and risk of reflux oesophagitis, Barrett's oesophagus and oesophageal adenocarcinoma (Number of patients, odds ratios and $95 \%$ confidence intervals)

\begin{tabular}{|c|c|c|c|c|c|c|c|c|}
\hline & \multirow[b]{2}{*}{ Controls $(n)$} & \multirow[b]{2}{*}{ Cases $(n)$} & \multicolumn{2}{|c|}{$\begin{array}{l}\text { Age- and energy- } \\
\text { adjusted }\end{array}$} & \multicolumn{2}{|c|}{$\begin{array}{l}\text { Multivariate- } \\
\text { adjusted }^{\star}\end{array}$} & \multicolumn{2}{|c|}{$\begin{array}{c}\text { Multivariate }+ \text { GOR- } \\
\text { adjusted } \dagger\end{array}$} \\
\hline & & & OR & $95 \% \mathrm{Cl}$ & OR & $95 \% \mathrm{Cl}$ & OR & $95 \% \mathrm{Cl}$ \\
\hline $\begin{array}{l}\text { Reflux oesophagitisł } \\
\text { Vitamin D }(\mu \mathrm{g} / \mathrm{d})\end{array}$ & 115 & 208 & & & & & & \\
\hline$<2.08$ & 36 & 70 & 1.00 & & 1.00 & & 1.00 & \\
\hline $2.08-<3.13$ & 41 & 76 & 0.94 & $0.52,1.71$ & 0.59 & $0.25,1.38$ & 0.59 & $0.25,1.39$ \\
\hline$\geq 3.13-9.2$ & 38 & 62 & 0.80 & $0.41,1.57$ & 0.64 & $0.25,1.63$ & 0.63 & $0.25,1.62$ \\
\hline $\begin{array}{c}P \text { for trend } \\
\mathrm{Ca}(\mathrm{mg} / \mathrm{d})\end{array}$ & & & \multicolumn{2}{|r|}{0.50} & \multicolumn{2}{|r|}{0.49} & \multicolumn{2}{|r|}{0.48} \\
\hline$<957$ & 38 & 78 & 1.00 & & 1.00 & & 1.00 & \\
\hline $957-<1316 \cdot 3$ & 39 & 76 & 0.87 & $0.47,1.61$ & 0.97 & $0.38,2 \cdot 51$ & 1.00 & $0.39,2.60$ \\
\hline$\geq 1316 \cdot 3-2796 \cdot 0$ & 38 & 54 & 0.37 & $0.17,0.80$ & 0.40 & $0.13,1.29$ & 0.41 & $0.13,1 \cdot 30$ \\
\hline$P$ for trend & & & \multicolumn{2}{|r|}{0.01} & \multicolumn{2}{|r|}{0.08} & \multicolumn{2}{|r|}{0.08} \\
\hline \multicolumn{9}{|l|}{ Dairy products $(\mathrm{g} / \mathrm{d}) \S$} \\
\hline$<285$ & 39 & 84 & 1.00 & & 1.00 & & 1.00 & \\
\hline $285-<472 \cdot 4$ & 38 & 64 & 0.77 & $0.44,1.36$ & 0.78 & $0.45,1.36$ & 0.70 & $0.32,1.53$ \\
\hline$\geq 472 \cdot 2-1032 \cdot 0$ & 38 & 60 & 0.77 & $0.43,1.36$ & 0.73 & $0.42,1.28$ & 0.69 & $0.31,1.51$ \\
\hline$P$ for trend & & & \multicolumn{2}{|r|}{0.39} & \multicolumn{2}{|r|}{0.29} & \multicolumn{2}{|r|}{0.37} \\
\hline Barrett's oesophagus & 252 & 212 & & & & & & \\
\hline \multicolumn{9}{|l|}{ Vitamin D $(\mu \mathrm{g} / \mathrm{d})$} \\
\hline$<2.05$ & 87 & 71 & 1.00 & & 1.00 & & 1.00 & \\
\hline $2.05-<3.0$ & 79 & 53 & 0.74 & $0.46,1.21$ & 0.75 & $0.40,1.43$ & 0.64 & $0.33,1.28$ \\
\hline$\geq 3 \cdot 0-9 \cdot 7$ & 86 & 88 & 1.05 & $0 \cdot 65,1 \cdot 70$ & $1 \cdot 24$ & $0 \cdot 68,2 \cdot 29$ & $1 \cdot 18$ & $0 \cdot 61,2 \cdot 29$ \\
\hline$P$ for trend & & & \multirow{2}{*}{\multicolumn{2}{|c|}{0.59}} & \multirow{2}{*}{\multicolumn{2}{|c|}{0.29}} & \multicolumn{2}{|r|}{0.33} \\
\hline $\mathrm{Ca}(\mathrm{mg} / \mathrm{d})$ & & & & & & & & \\
\hline$<929 \cdot 3$ & 85 & 66 & 1.00 & & 1.00 & & 1.00 & \\
\hline $929 \cdot 3-<1262 \cdot 0$ & 83 & 65 & 0.85 & $0.52,1.38$ & 0.68 & $0.35,1.33$ & 0.65 & $0.32,1.32$ \\
\hline$\geq 1262 \cdot 0-2796 \cdot 0$ & 84 & 81 & 0.91 & $0.52,1.68$ & 0.80 & $0 \cdot 36,1 \cdot 77$ & 0.66 & $0.28,1.56$ \\
\hline$P$ for trend & & & & 0.78 & & 0.71 & & 0.43 \\
\hline Dairy products $(\mathrm{g} / \mathrm{d}) \S$ & & & & & & & & \\
\hline$<281.5$ & 84 & 59 & 1.00 & & 1.00 & & 1.00 & \\
\hline $281.5-<493.2$ & 84 & 84 & 1.43 & $0.91,2.25$ & 1.63 & $0.91,2.92$ & 1.83 & $0.97,3.42$ \\
\hline$\geq 493 \cdot 2-999.9$ & 84 & 69 & $1 \cdot 21$ & $0.76,1.92$ & 1.45 & $0 \cdot 80,2 \cdot 64$ & 1.94 & $1 \cdot 01,3 \cdot 71$ \\
\hline$P$ for trend & & & & 0.63 & & 0.36 & & 0.09 \\
\hline Oesophageal adenocarcinoma & 252 & 218 & & & & & & \\
\hline Vitamin D $(\mu \mathrm{g} / \mathrm{d})$ & & & & & & & & \\
\hline$<2.05$ & 87 & 48 & 1.00 & & 1.00 & & 1.00 & \\
\hline $2.05-<3.0$ & 79 & 68 & 1.43 & $0.87,2 \cdot 34$ & $1 \cdot 11$ & $0 \cdot 56,2 \cdot 21$ & 1.00 & $0.50,2.01$ \\
\hline$\geq 3 \cdot 0-9 \cdot 7$ & 86 & 102 & 1.85 & $1 \cdot 12,3.05$ & 1.99 & $1.03,3.86$ & 1.92 & $0.98,3.76$ \\
\hline$P$ for trend & & & & 0.02 & & 0.02 & & 0.03 \\
\hline $\mathrm{Ca}(\mathrm{mg} / \mathrm{d})$ & & & & & & & & \\
\hline$<929 \cdot 3$ & 85 & 62 & 1.00 & & 1.00 & & 1.00 & \\
\hline $929 \cdot 3-<1262 \cdot 0$ & 83 & 73 & 1.06 & $0.66,1 \cdot 73$ & 0.97 & $0.49,1.92$ & 0.94 & $0.47,1.88$ \\
\hline$\geq 1262 \cdot 0-2796 \cdot 0$ & 84 & 83 & 1.00 & $0.57,1.75$ & 0.88 & $0.38,2.03$ & 0.80 & $0.34,1.87$ \\
\hline$P$ for trend & & & & 0.95 & & 0.75 & & 0.59 \\
\hline Dairy products $(\mathrm{g} / \mathrm{d}) \S$ & & & & & & & & \\
\hline$<281.5$ & 84 & 60 & 1.00 & & 1.00 & & 1.00 & \\
\hline $281 \cdot 5-<493.2$ & 84 & 71 & $1 \cdot 17$ & $0.74,1 \cdot 86$ & $1 \cdot 30$ & $0 \cdot 70,2 \cdot 42$ & 1.34 & $0.71,2.52$ \\
\hline$\geq 493.2-999.9$ & 84 & 87 & 1.50 & $0.96,2.35$ & 1.67 & $0.91,3.06$ & 1.89 & $1.02,3.50$ \\
\hline$P$ for trend & & & & 0.07 & & 0.10 & & 0.04 \\
\hline
\end{tabular}

GOR, gastro-oesophageal reflux.

${ }^{*}$ Multivariate adjustments: age (years); sex; energy intake (kJ/d); smoking status (current/previous/never); BMI 5 years before the interview; education (years); occupation (manual/non-manual); alcohol (g/d); regular non-steroidal anti-inflammatory drug use (ever/never: ever, weekly use for at least 6 months' duration); Helicobacter pylori infection (seropositive/seronegative); energy-adjusted glycaemic index intake (units/4184 kJ per d); energy-adjusted saturated fat intake (g/4184 kJ per d); location (Northern Ireland/Republic of Ireland).

† GOR symptoms experienced at least once weekly or $>50$ times/year $>5$ years before the interview date.

$\ddagger$ Analysis limited to Northern Ireland controls only.

$\S$ Dairy product intake defined as milk, yogurt, Greek yogurt, cheese, processed cheese and cottage cheese.

Nevertheless, BMI is a well-established correlate of vitamin D status, with obesity linked with lower circulating 25-hydroxyvitamin $\mathrm{D}$ concentrations ${ }^{(31-33)}$, which makes the present findings even more surprising. This association is possibly confounded by lower outdoor physical activity levels among overweight individuals. Similar converse results were observed for $H$. pylori positivity, which is a highly significant protective factor in oesophageal adenocarcinoma development ${ }^{(26)}$, and yet we observed a heightened risk among H. pylori-positive individuals with the highest vitamin D intakes. A significant positive correlation between serum vitamin $\mathrm{D}$ concentrations and $H$. pylori status has been 
Table 3. Vitamin D-related foods and risk of oesophageal adenocarcinoma (OAC)

(Number of patients, odds ratios and $95 \%$ confidence intervals)

\begin{tabular}{|c|c|c|c|c|c|c|c|c|}
\hline \multirow[b]{2}{*}{ Food group } & \multirow[b]{2}{*}{ Controls $(n)$} & \multirow[b]{2}{*}{ OAC $(n)$} & \multicolumn{2}{|c|}{$\begin{array}{l}\text { Age- and energy- } \\
\text { adjusted }\end{array}$} & \multicolumn{2}{|c|}{ Multivariate-adjusted* } & \multicolumn{2}{|c|}{$\begin{array}{c}\text { Multivariate- } \\
\text { adjusted + GOR } \dagger\end{array}$} \\
\hline & & & OR & $95 \% \mathrm{Cl}$ & OR & $95 \% \mathrm{Cl}$ & OR & $95 \% \mathrm{Cl}$ \\
\hline \multicolumn{9}{|l|}{ Oily fish (portions/week) } \\
\hline 0 & 85 & 82 & 1.00 & & 1.00 & & 1.00 & \\
\hline 0.5 & 101 & 76 & 0.74 & $0.48,1.13$ & 0.80 & $0.45,1.44$ & 0.82 & $0.45,1.4 \varepsilon$ \\
\hline$\geq 1$ & 66 & 60 & 0.90 & $0.56,1.43$ & $1 \cdot 21$ & $0.65,2.27$ & $1 \cdot 20$ & $0.63,2 \cdot 2 \varepsilon$ \\
\hline$P$ for trend & & & \multicolumn{2}{|r|}{0.58} & \multicolumn{2}{|r|}{0.60} & \multicolumn{2}{|r|}{0.63} \\
\hline \multicolumn{9}{|l|}{ Eggs (portions/week) } \\
\hline$<1.5$ & 90 & 72 & 1.00 & & 1.00 & & 1.00 & \\
\hline $1.5-<3.5$ & 82 & 60 & 0.88 & $0.56,1.40$ & 1.05 & $0.57,1.93$ & 1.00 & $0.54,1.86$ \\
\hline$\geq 3.5$ & 80 & 86 & 1.32 & $0.85,2.04$ & $1 \cdot 19$ & $0.65,2.17$ & $1 \cdot 18$ & $0.64,2 \cdot 1 \varepsilon$ \\
\hline$P$ for trend & & & \multicolumn{2}{|r|}{0.11} & \multicolumn{2}{|r|}{0.57} & \multicolumn{2}{|r|}{0.55} \\
\hline \multicolumn{9}{|l|}{ Margarine (portions/week) } \\
\hline 0 & 108 & 101 & 1.00 & & 1.00 & & 1.00 & \\
\hline Any (median 17.5 tsp/week) & 144 & 117 & 0.92 & $0.64,1.33$ & 1.09 & $0.66,1 \cdot 80$ & $1 \cdot 10$ & $0.66,1.83$ \\
\hline$P$ for trend & & & \multicolumn{2}{|r|}{0.66} & \multicolumn{2}{|r|}{0.72} & \multicolumn{2}{|r|}{0.71} \\
\hline \multicolumn{9}{|l|}{ Total red meat (portions/week)‡ } \\
\hline$<4.5$ & 64 & 48 & 1.00 & & 1.00 & & 1.00 & \\
\hline $4.5-<7$ & 101 & 85 & $1 \cdot 12$ & $0.70,1.80$ & 1.12 & $0.58,2.16$ & 1.14 & $0.58,2.22$ \\
\hline$\geq 7$ & 87 & 85 & 1.29 & $0.80,2.09$ & 1.03 & $0.53,2.01$ & 1.03 & $0.52,2.03$ \\
\hline$P$ for trend & & & \multicolumn{2}{|r|}{0.29} & \multicolumn{2}{|r|}{0.99} & \multicolumn{2}{|r|}{0.99} \\
\hline \multicolumn{9}{|l|}{ Liver (portions/week) } \\
\hline 0 & 192 & 135 & 1.00 & & 1.00 & & 1.00 & \\
\hline Any (median 0.5 ) & 60 & 83 & 1.94 & $1 \cdot 30,2 \cdot 89$ & 1.73 & $1.01,2.97$ & 1.67 & $0.97,2.8 \mathrm{~s}$ \\
\hline$P$ for trend & & & & 0.001 & & 0.05 & & 0.07 \\
\hline
\end{tabular}

GOR, gastro-oesophageal reflux; tsp, teaspoon.

* Multivariate adjustments: age (years); sex; energy intake (kJ/d); smoking status (current/previous/never); BMI 5 years before the interview (kg/m²); education (years); occupation (manual/non-manual); alcohol (g/d); regular non-steroidal anti-inflammatory drug use (weekly use for at least 6 months' duration); location (Northern Ireland/Republic of Ireland); Helicobacter pylori infection (seropositive/seronegative); energy-adjusted saturated fat intake (g/4184 kJ per d); energy-adjusted glycaemic index intake (units/4184 kJ per d).

† GOR symptoms experienced at least once weekly or $>50$ times/year $>5$ years before the interview date.

$\ddagger$ Total red meat intake: beef + pork + ham + lamb + liver.

reported in a small cross-sectional study of end-stage renal disease patients ${ }^{(34)}$, suggesting that vitamin $\mathrm{D}$ may modulate H. pylori activity, but this hypothesis requires considerable investigation to elucidate any potential pathways. A history of smoking also appeared to increase oesophageal adenocarcinoma risk for high vitamin D consumers. Correspondingly, results from a pooled sample of upper gastrointestinal cancers illustrated a significant reduced risk for never smokers with low vitamin D status ${ }^{(35)}$. Given the lack of statistical significance in interaction tests in the present study though, it is likely that the associations observed in stratified analysis are subject to differential misclassification bias and should be interpreted with caution.

Finally, it may be that nutrient-gene interactions are explaining the associations observed. The vitamin D receptor has been demonstrated to regulate bile acid metabolism in vivo ${ }^{(36)}$, and bile acids are known to induce DNA damage that will ultimately encourage malignant growth in human gastrointestinal tissue ${ }^{(37)}$. Therefore, the association between vitamin $\mathrm{D}$ receptor polymorphisms and oesophageal adenocarcinoma risk will provide a useful insight into potential mechanisms involved.

The direct association between dietary vitamin D and oesophageal adenocarcinoma risk is perhaps not entirely surprising though. An international consortium has recently published their findings from a Vitamin D Pooling Project of
Rarer Cancers ${ }^{(38)}$. Combining data of circulating 25-hydroxyvitamin D concentrations, the research team found that high vitamin D status significantly doubled the risk of pancreatic cancer ${ }^{(39)}$, while there was no evidence of an association between 25-hydroxyvitamin D concentrations and the combined risk of oesophageal and gastric cancers, which included 104 oesophageal adenocarcinomas ${ }^{(35)}$. The research team deemed that 'careful consideration' should be taken when recommending healthy individuals to increase their vitamin $\mathrm{D}$ intake ${ }^{(39)}$. Importantly, these findings do not negate the proven inverse association between vitamin $\mathrm{D}$ and colorectal cancer and adenoma risk ${ }^{(16,17)}$, but suggest that more appropriate targeting of individuals is required for advising an increased intake of vitamin $\mathrm{D}$ from foods or conduction of supplementation trials in future.

The present study has several strengths, including its population-based design, which maximises the generalisability of the findings, and a large number of cases included. A wide variety of confounding variables were able to be accounted for in regression analyses, including $H$. pylori infection, which was not available in the Cohort Consortium Vitamin D Pooling Project ${ }^{(35)}$. However, there is the possibility that some residual confounding may be occurring in these analyses. We do not underestimate the key limitation that this study examines vitamin D intake only and does not account for additional consumption via supplements, nor serum 
Table 4. Stratified analysis of vitamin D and oesophageal adenocarcinoma risk by BMI, Helicobacter pylori and smoking categories* (Number of patients, odds ratios and $95 \%$ confidence intervals)

\begin{tabular}{|c|c|c|c|c|c|c|c|c|c|c|c|c|}
\hline \multirow{2}{*}{$\begin{array}{l}\text { Oesophageal } \\
\text { adenocarcinoma }\end{array}$} & \multirow{2}{*}{$\begin{array}{l}\text { Cases/ } \\
\text { controls }(n)\end{array}$} & \multicolumn{2}{|c|}{$\begin{array}{c}\mathrm{BMI} \\
\left(<25 \mathrm{~kg} / \mathrm{m}^{2}\right) \dagger\end{array}$} & \multirow{2}{*}{$\begin{array}{l}\text { Cases/ } \\
\text { controls }(n)\end{array}$} & \multicolumn{2}{|c|}{$\begin{array}{c}\text { BMI } \\
\left(\geq 25 \mathrm{~kg} / \mathrm{m}^{2}\right) \dagger\end{array}$} & \multirow{2}{*}{$\begin{array}{l}\text { Cases/ } \\
\text { controls }(n)\end{array}$} & \multicolumn{2}{|c|}{$\begin{array}{l}\text { H. pylorif } \\
\text { negative }\end{array}$} & \multirow{2}{*}{$\begin{array}{l}\text { Cases/ } \\
\text { controls }(n)\end{array}$} & \multicolumn{2}{|c|}{$\begin{array}{l}\text { H. pylori } \\
\text { positiveł }\end{array}$} \\
\hline & & OR & $95 \% \mathrm{Cl}$ & & OR & $95 \% \mathrm{Cl}$ & & OR & $95 \% \mathrm{Cl}$ & & OR & $95 \% \mathrm{Cl}$ \\
\hline \multicolumn{13}{|l|}{ Vitamin $D(\mu \mathrm{g} / \mathrm{d})$} \\
\hline$<2.05$ & $12 / 25$ & 1.00 & & $36 / 62$ & 1.00 & & $25 / 33$ & 1.00 & & $19 / 51$ & 1.00 & \\
\hline $2.05-<3.0$ & $21 / 21$ & 1.38 & $0.34,5.59$ & $47 / 58$ & 1.01 & $0.45,2.25$ & $34 / 41$ & 0.57 & $0.21,1.49$ & $26 / 35$ & $2 \cdot 32$ & $0.78,6.88$ \\
\hline$\geq 3 \cdot 0$ & $17 / 31$ & 0.91 & $0.22,3.87$ & $85 / 55$ & $2 \cdot 49$ & $1 \cdot 16,5 \cdot 37$ & $47 / 30$ & 0.98 & $0.35,2.77$ & $51 / 54$ & 3.78 & $1.44,9.91$ \\
\hline$P$ for trend & & 0.76 & & & 0.008 & & & 0.72 & & & 0.008 & \\
\hline \multirow{4}{*}{$\begin{array}{l}\text { per } 1 \mu \mathrm{g} / \mathrm{d} \\
\text { increment }\end{array}$} & $50 / 77$ & 0.95 & $0.67,1.35$ & $168 / 175$ & 1.32 & $1.06,1.65$ & 106/104 & 1.04 & $0 \cdot 78,1 \cdot 38$ & $96 / 140$ & 1.33 & $1 \cdot 04,1 \cdot 70$ \\
\hline & \multicolumn{6}{|c|}{$P$ for interaction 0.50} & \multirow{2}{*}{\multicolumn{6}{|c|}{$P$ for interaction 0.57}} \\
\hline & \multirow{2}{*}{$\begin{array}{l}\text { Cases/ } \\
\text { controls }(n)\end{array}$} & \multicolumn{2}{|c|}{ Never smokers§ } & \multirow{2}{*}{$\begin{array}{l}\text { Cases/ } \\
\text { controls }(n)\end{array}$} & \multicolumn{2}{|c|}{ Previous smokers§ } & & & & & & \\
\hline & & OR & $95 \% \mathrm{Cl}$ & & OR & $95 \% \mathrm{Cl}$ & $\begin{array}{l}\text { Cases/ } \\
\text { controls }(n)\end{array}$ & OR & $95 \% \mathrm{Cl}$ & $\begin{array}{l}\text { Cases/ } \\
\text { controls }(n)\end{array}$ & OR & $95 \% \mathrm{Cl}$ \\
\hline $\begin{array}{l}\text { Vitamin D } \\
\text { per } 1 \mu \mathrm{g} / \mathrm{d} \\
\text { increment }\end{array}$ & $44 / 100$ & $1 \cdot 16$ & $0 \cdot 80,1 \cdot 70$ & $94 / 101$ & 1.46 & $1 \cdot 04,2 \cdot 05$ & $75 / 44$ & 1.04 & $0.77,1.40$ & $169 / 145$ & $1 \cdot 23$ & $0.99,1.52$ \\
\hline
\end{tabular}

*Multivariate adjustments: age (years); sex; energy intake (kJ/d); education (years); occupation (manual/non-manual); alcohol (g/d); regular non-steroidal anti-inflammatory drug use (weekly use for at least 6 months' duration); "Multivariate adjustments: age (years); sex; energy intake (kJ/d); education (years); occupation (manual/non-manual); alcohol (g/d); regular non-steroidal anti-inflamme
location (Northern Ireland/Republic of Ireland); energy-adjusted saturated fat intake (g/4184 kJ per d); energy-adjusted glycaemic index intake (units/4184 kJ per d).

$\dagger \mathrm{\dagger MI}$ analysis further adjusted for $H$. pylori infection (seropositive/seronegative) and smoking status (current/previous/never).

$\ddagger H$. pylori analysis further adjusted for BMI 5 years before the interview and smoking status (current/previous/never).

$\S$ Smoking status analysis further adjusted for BMI 5 years before the interview and H. pylori infection (seropositive/seronegative). 
25-hydroxyvitamin D concentrations, and therefore does not account for cutaneous vitamin D production from sunlight. Indeed, recent data from the USA confirm that dietary vitamin $\mathrm{D}$ is only partially correlated with serum 25-hydroxyvitamin D concentrations and therefore does not fully reflect vitamin D status $^{(40)}$. Unfortunately, it would be inappropriate to assess vitamin $\mathrm{D}$ status in the present study due to the possibility of reverse causation, and it would be useful for serum 25hydroxyvitamin $\mathrm{D}$ and oesophageal adenocarcinoma risk to be assessed in future prospective studies. It is possible that dietary recall bias among the oesophageal adenocarcinoma cases or dietary measurement error from the FFQ utilised may be explaining the association observed. The potential for selection bias may also have been introduced by the lower response rate among our population-based controls compared with cases. However, mean vitamin D intakes observed in the present study are in line with those measured in the representative North/South Ireland Food Consumption Survey of $3.7 \mu \mathrm{g} / \mathrm{d}^{(41)}$, as were Ca intakes ${ }^{(42)}$. Moreover, supplements were found to contribute to only $6 \cdot 2$ and $11.4 \%$ of vitamin $\mathrm{D}$ intakes by men and women in the same nationally representative survey, respectively ${ }^{(41)}$. Vitamin D insufficiency is highly prevalent in healthy Irish adults ${ }^{(43)}$, prompting calls for a nationwide fortification or supplementation programme. Yet, it cannot be ignored that certain population subgroups may be at risk of harm if advice to increase vitamin D intake from foods was implemented on a widespread basis. These findings provide specific evidence for a research direction call on vitamin $\mathrm{D}$ to identify population groups that may be at risk with vitamin D intake modification. Many Barrett's oesophagus patients remain asymptomatic and therefore undiagnosed $^{(44)}$, and so any necessary restrictions should perhaps be extended to regular sufferers of gastro-oesophageal reflux symptoms. However, further research is required to confirm the direct association between vitamin D intake and oesophageal adenocarcinoma risk and to elucidate the potential mechanism involved.

In conclusion, intake of vitamin D-containing foods was directly associated with oesophageal adenocarcinoma risk in this Irish population but was not related to the risk of its precursor conditions. $\mathrm{Ca}$ and dairy product intakes did appear to contribute to the development of these oesophageal lesions.

\section{Acknowledgements}

The authors' contributions were as follows: H. G. M. performed the statistical analysis and drafted the manuscript; L. J. M. was the original grant holder for the FINBAR study; L. A. A. recruited the study participants and collected the study data; M. M. C. provided guidance on the statistical analysis and nutritional knowledge. All authors contributed to the writing and editing of the final manuscript. The authors state that there are no conflicts of interest. The FINBAR study group members include L. J. M., L. A. A. (Queen's University Belfast), B. T. Johnston, R. G. P. Watson, J. McGuigan, H. R. Ferguson (Belfast Health and Social Care Trust, Belfast, County Antrim, UK), S. J. Murphy (St Vincent's Hospital Dublin, Dublin, Ireland), J. V. Reynolds (St James' Hospital,
Dublin, Ireland) and H. Comber (National Cancer Registry of Ireland, Cork, Ireland). We appreciate the contributions made by the study participants, their families and all who assisted with the study, particularly the Northern Ireland Cancer Registry and the National Cancer Registry Cork. We also thank Dr Christian Abnet (National Cancer Institute, Bethesda, MD, USA) for his helpful comments on the manuscript. The present study was supported by funding from the Ulster Cancer Foundation, the Northern Ireland R\&D office and the Health Research Board.

\section{References}

1. Lepage C, Rachet B, Jooste V, et al. (2008) Continuing rapid increase in esophageal adenocarcinoma in England and Wales. Am J Gastroenterol 103, 2694-2699.

2. El-Serag HB (2007) Time trends of gastroesophageal reflux disease: a systematic review. Clin Gastroenterol Hepatol 5 , $17-26$.

3. Shaheen NJ \& Richter JE (2009) Barrett's oesophagus. Lancet 373, 850-861.

4. Wood RK \& Yang YX (2008) Barrett's esophagus in 2008: an update. Keio J Med 57, 132-138.

5. Yousef F, Cardwell C, Cantwell MM, et al. (2008) The incidence of esophageal cancer and high-grade dysplasia in Barrett's esophagus: a systematic review and metaanalysis. Am J Epidemiol 168, 237-249.

6. Thomas T, Abrams KR, De Caestecker JS, et al. (2007) Meta analysis: cancer risk in Barrett's oesophagus. Aliment Pharmacol Ther 26, 1465-1477.

7. Terry P, Lagergren J, Wolk A, et al. (2000) Reflux-inducing dietary factors and risk of adenocarcinoma of the esophagus and gastric cardia. Nutr Cancer 38, 186-191.

8. El-Serag HB, Satia JA \& Rabeneck L (2005) Dietary intake and the risk of gastro-oesophageal reflux disease: a cross sectional study in volunteers. Gut 54, 11-17.

9. Anderson LA, Watson RG, Murphy SJ, et al. (2007) Risk factors for Barrett's oesophagus and oesophageal adenocarcinoma: results from the FINBAR study. World $J$ Gastroenterol 13, 1585-1594.

10. Kubo A, Levin TR, Block G, et al. (2008) Dietary antioxidants, fruits, and vegetables and the risk of Barrett's esophagus. Am J Gastroenterol 103, 1614-1623, quiz 1624.

11. Thompson OM, Beresford SA, Kirk EA, et al. (2009) Vegetable and fruit intakes and risk of Barrett's esophagus in men and women. Am J Clin Nutr 89, 890-896.

12. World Cancer Research Fund/American Institute for Cancer Research (2007) Food, Nutrition, Physical Activity, and the Prevention of Cancer: A Global Perspective. Washington DC: AICR.

13. Mulholland HG, Cantwell MM, Anderson LA, et al. (2009) Glycemic index, carbohydrate and fiber intakes and risk of reflux esophagitis, Barrett's esophagus, and esophageal adenocarcinoma. Cancer Causes Control 20, 279-288.

14. Giovannucci E (2008) Vitamin D and cancer incidence in the Harvard cohorts. Ann Epidemiol 19, 84-88.

15. Dusso AS, Brown AJ \& Slatopolsky E (2005) Vitamin D. Am J Physiol Renal Physiol 289, F8-28.

16. Gorham ED, Garland CF, Garland FC, et al. (2007) Optimal vitamin D status for colorectal cancer prevention: a quantitative meta analysis. Am J Prev Med 32, 210-216.

17. Wei MY, Garland CF, Gorham ED, et al. (2008) Vitamin D and prevention of colorectal adenoma: a meta-analysis. Cancer Epidemiol Biomarkers Prev 17, 2958-2969. 
18. Stolzenberg-Solomon RZ, Vieth R, Azad A, et al. (2006) A prospective nested case-control study of vitamin D status and pancreatic cancer risk in male smokers. Cancer Res 66, 10213-10219.

19. Abnet CC, Chen W, Dawsey SM, et al. (2007) Serum 25(OH)vitamin D concentration and risk of esophageal squamous dysplasia. Cancer Epidemiol Biomarkers Prev 16, 1889-1893.

20. Chen W, Dawsey SM, Qiao YL, et al. (2007) Prospective study of serum $25(\mathrm{OH})$-vitamin D concentration and risk of oesophageal and gastric cancers. Br J Cancer 97, 123-128.

21. Davis CD (2008) Vitamin D and cancer: current dilemmas and future research needs. Am J Clin Nutr 88, 565S-569S.

22. Anderson LA, Johnston BT, Watson RG, et al. (2006) Nonsteroidal anti-inflammatory drugs and the esophageal inflammation-metaplasia-adenocarcinoma sequence. Cancer Res 66, 4975-4982.

23. Nayar DS \& Vaezi MF (2004) Classifications of esophagitis: who needs them? Gastrointest Endosc 60, 253-257.

24. Day N, Oakes S, Luben R, et al. (1999) EPIC-Norfolk: study design and characteristics of the cohort. European Prospective Investigation of Cancer. $B r J$ Cancer 80, Suppl. 1, 95-103.

25. Harrington KE, Robson PJ, Kiely M, et al. (2001) The North/ South Ireland Food Consumption Survey: survey design and methodology. Public Health Nutr 4, 1037-1042.

26. Anderson LA, Murphy SJ, Johnston BT, et al. (2008) Relationship between Helicobacter pylori infection and gastric atrophy and the stages of the oesophageal inflammation, metaplasia, adenocarcinoma sequence: results from the FINBAR case-control study. Gut 57, 734-739.

27. Mayne ST, Risch HA, Dubrow R, et al. (2001) Nutrient intake and risk of subtypes of esophageal and gastric cancer. Cancer Epidemiol Biomarkers Prev 10, 1055-1062.

28. Navarro Silvera SA, Mayne ST, Risch H, et al. (2008) Food group intake and risk of subtypes of esophageal and gastric cancer. Int J Cancer 123, 852-860.

29. Garland CF, Garland FC, Gorham ED, et al. (2006) The role of vitamin D in cancer prevention. Am J Public Health 96, $252-261$.

30. Koohdani F, Baghdadchi J, Sasani F, et al. (2008) Effects of vitamin D in lung, stomach, esophagus and testis tissues following administration of urethane in balb/c mice. Saudi Med J 29, 340-344.

31. Kull M, Kallikorm R \& Lember M (2009) Body mass index determines sunbathing habits: implications on vitamin D levels. Intern Med J 39, 256-258.
32. Konradsen S, Ag H, Lindberg F, et al. (2008) Serum 1,25dihydroxy vitamin $\mathrm{D}$ is inversely associated with body mass index. Eur J Nutr 47, 87-91.

33. Lagunova Z, Porojnicu AC, Lindberg F, et al. (2009) The dependency of vitamin $D$ status on body mass index, gender, age and season. Anticancer Res 29, 3713-3720.

34. Nasri H \& Baradaran A (2007) The influence of serum 25hydroxy vitamin D levels on Helicobacter pylori infections in patients with end-stage renal failure on regular hemodialysis. Saudi J Kidney Dis Transpl 18, 215-219.

35. Abnet CC, Chen Y, Chow WH, et al. (2010) Circulating 25-hydroxyvitamin D and risk of esophageal and gastric cancer: Cohort Consortium Vitamin D Pooling Project of Rarer Cancers. Am J Epidemiol 172, 94-106.

36. Nishida S, Ozeki J \& Makishima M (2009) Modulation of bile acid metabolism by 1alpha-hydroxyvitamin $\mathrm{D}_{3}$ administration in mice. Drug Metab Dispos 37, 2037-2044.

37. Bernstein H, Bernstein C, Payne CM, et al. (2005) Bile acids as carcinogens in human gastrointestinal cancers. Mutat Res 589, $47-65$.

38. Helzlsouer KJ \& VDPP Steering Committee (2010) Overview of the Cohort Consortium Vitamin D Pooling Project of Rarer Cancers. Am J Epidemiol 172, 4-9.

39. Stolzenberg-Solomon RZ, Jacobs EJ, Arslan AA, et al. (2010) Circulating 25-hydroxyvitamin D and risk of pancreatic cancer: Cohort Consortium Vitamin D Pooling Project of Rarer Cancers. Am J Epidemiol 172, 81-93.

40. Millen AE, Wactawski-Wende J, Pettinger M, et al. (2010) Predictors of serum 25-hydroxyvitamin D concentrations among postmenopausal women: the Women's Health Initiative Calcium plus Vitamin D clinical trial. Am J Clin Nutr 91, 1324-1335.

41. O'Brien MM, Kiely M, Harrington KE, et al. (2001) The North/South Ireland Food Consumption Survey: vitamin intakes in 18-64-year-old adults. Public Health Nutr 4, 1069-1079.

42. Hannon EM, Kiely M, Harrington KE, et al. (2001) The North/South Ireland Food Consumption Survey: mineral intakes in 18-64-year-old adults. Public Health Nutr 4, $1081-1088$.

43. O'Sullivan M, Nic Suibhne T, Cox G, et al. (2008) High prevalence of vitamin $\mathrm{D}$ insufficiency in healthy Irish adults. Ir J Med Sci 177, 131-134.

44. Fitzgerald RC (2004) Review Article: Barrett's oesophagus and associated adenocarcinoma - a UK perspective. Aliment Pharmacol Ther 20, Suppl. 8, 45-49. 